\title{
Mecânica Clássica - Momento linear e trabalho
}

\author{
Mario Cezar Bertin ${ }^{1}$ \\ ${ }^{1}$ Instituto de Física, Universidade Federal da Bahia
}

April 26, 2021

\begin{abstract}
Neste texto, vamos tratar do postulado que tem a interpretação da conservação do momento linear de um sistema de partículas isolado. Veremos como este postulado dá origem à segunda e à terceira leis de Newton.
\end{abstract}

\section{A conservação do movimento}

Nas seções passadas, vimos como dois postulados fundamentais estabelecem a dinâmica do movimento de uma partícula. O postulado 5 é uma releitura da primeira lei de Newton. Para nossos propósitos, ela é importante para a definição dos referenciais inerciais. Esta definição difere da apresentação usual da primeira lei em um aspecto importante. Substitui o conceito de força (ou ausência de), por um conceito mais preciso, o de partículas isoladas. Neste contexto, uma partícula isolada é aquela longe o suficiente de qualquer outra partícula (ou campo), que possa com ela interagir. De fato, a existência de um referencial inercial implica na existência de infinitos referenciais inerciais, todos medindo uma partícula isolada como aquela em movimento retilíneo uniforme. Dizemos, também, que tal partícula é livre, ou inercial.

O postulado 6 estabelece a conservação da energia total de um sistema isolado. Com estes postulados, somos capazes de tratar sistemas unidimensionais, ou seja, sistemas com apenas um graude liberdade. Foram os casos da partícula livre, da partícula sujeita a um potencial linear na posição e da partícula sujeita ao potencial quadrático. Contudo, problemas surgem quando temos dois ou mais graus de liberdade, pois a conservação da energia não é suficiente para determinar univocamente a dinâmica de sistemas multi-dimensionais.

Este problema tem relação com a definição e resolução, mais precisamente da integrabilidade, de equações diferenciais ordinárias, que de fato descrevem a dinâmica desses sistemas.

Introduzimos, assim, o postulado

\section{Postulado 7 - Princípio da conservação do movimento:}

Sejam $n$ partículas que interagem ente si, mas são isoladas em conjunto, observadas por um observador inercial. Seja $\mathbf{v}_{I}(t)$ o campo de velocidades da partícula $I$, sendo $I=1, \cdots, n$. Então:

1. Existe uma combinação linear única e positiva definida das velocidades tais que o vetor resultante é uma constante no tempo, ou seja,

$$
\exists m_{I} \in \mathbb{R}_{+}: \sum_{I} m_{I} \mathbf{v}_{I}(t)=\mathbf{p}, \quad \text { e } \quad \frac{d \mathbf{p}}{d t}=0 .
$$

As constantes $m_{I}$ são independentes do tempo, do sistema referencial escolhido e da natureza da interação. Dependem apenas da natureza das partículas. 
Com a proposição acima, podemos prosseguir com as seguintes definições:

Massa e momento linear: A constante $m_{I}$ é definida como a massa referente à partículas $I$. $\mathrm{O}$ vetor $\mathbf{p}$ é denominado quantidade de movimento, momento linear, ou simplesmente momento do sistema.

Vamos considerar, por simplicidade, o exemplo de duas partículas isoladas. Temos

$$
\mathbf{p}=m_{1} \mathbf{v}_{1}+m_{2} \mathbf{v}_{2} .
$$

Neste caso, definimos o momento de cada partícula como

$$
\mathbf{p}_{1}=m_{1} \mathbf{v}_{1}, \quad \mathbf{p}_{2}=m_{2} \mathbf{v}_{2},
$$

de modo que

$$
\mathbf{p}=\mathbf{p}_{1}+\mathbf{p}_{2} \text {. }
$$

Evidentemente, se as velocidades são vetores por rotações, os momentos também o são. Se $\mathbf{p}$ é constante, temos

ou seja,

$$
\frac{d \mathbf{p}}{d t}=0 \quad \Longrightarrow \quad \frac{d}{d t}\left(m_{1} \mathbf{v}_{1}+m_{2} \mathbf{v}_{2}\right)=0
$$

$$
m_{1} \mathbf{a}_{1}+m_{2} \mathbf{a}_{2}=0
$$

Voltando ao sistema de $\mathrm{n}$ partículas, as expressões anteriores resultam em

$$
\mathbf{p}=\sum_{I} \mathbf{p}_{I}, \quad \mathbf{p}_{I}=m_{I} \mathbf{v}_{I},
$$

bem como

$$
\frac{d \mathbf{p}}{d t}=0 \quad \Longrightarrow \quad \sum_{I} m_{I} \mathbf{a}_{I}=0
$$

\section{Força}

Da equação (??), vemos que a conservação do momento implica em que a soma da massa vezes a aceleração de cada partícula deve ser nula. Podemos definir

Força: A força que age sobre uma partícula $I$ de momento linear $\mathbf{p}_{I}$ e massa $m_{I}$ constante é definida pela expressão

$$
\mathbf{F}_{I}=\frac{d \mathbf{p}_{I}}{d t}=m_{I} \mathbf{a}_{I}
$$

Note que $\mathbf{F}$ é também um vetor por rotações. Dessa forma, a conservação do momento implica em $\sum_{I} \mathbf{F}_{I}=$ 0(5)para um sistema de partículas isolado. A definição (??) é equivalente à segunda lei de Newton.

Voltando ao sistema de duas partículas, (??) resulta em

$$
\mathbf{F}_{1}=-\mathbf{F}_{2},
$$

ou seja, a força que a partícula 2 exerce sobre a partícula 1 é igual ao negativo da força que a partícula 1 exerce sobre a partícula 2. Esta é a expressão matemática da terceira lei de Newton na forma forte. 


\section{Novamente, a Energia}

Vamos tomar o caso de uma partícula de massa $m$ sobre a qual age uma força $\mathbf{F}$. Neste caso, a dinâmica é dada pela

$$
\mathbf{F}=m \mathbf{a}=m \dot{\mathbf{v}}=m \ddot{\mathbf{x}}
$$

ou seja, conhecida a força $\mathbf{F}=\mathbf{F}(t, \mathbf{x}, \dot{\mathbf{x}})$, (??) torna-se um sistema de três equações diferenciais ordinárias para o vetor posição.

Vamos tomar o produto escalar de (??) por $\mathbf{v}$, que resulta em

$$
\mathbf{F} \cdot \mathbf{v}=m \ddot{\mathbf{x}} \cdot \mathbf{v}=m \ddot{\mathbf{x}} \cdot \dot{\mathbf{x}}
$$

Note que

$$
\frac{d}{d t}\left(\frac{1}{2} m \dot{\mathbf{x}} \cdot \dot{\mathbf{x}}\right)=\frac{1}{2} m \frac{d}{d t}(\dot{\mathbf{x}} \cdot \dot{\mathbf{x}})=m \ddot{\mathbf{x}} \cdot \dot{\mathbf{x}}
$$

portanto,

$$
\mathbf{F} \cdot \dot{\mathbf{x}}=\frac{d}{d t}\left(\frac{1}{2} m \dot{\mathbf{x}} \cdot \dot{\mathbf{x}}\right)=\frac{d}{d t}\left(\frac{1}{2} m \dot{\mathbf{x}}^{2}\right)
$$

Aqui, reconhecemos a quantidade $K=\frac{1}{2} m \dot{\mathbf{x}}^{2}$ como a energia cinética.

Da equação (??) vemos que, se a força é ortogonal à velocidade, a energia cinética se conserva. Ou seja,

$$
\mathbf{F} \cdot \mathbf{v}=0 \quad \Longrightarrow \quad \frac{d K}{d t}=0
$$

Este caso inclui o da partícula livre, em que $\mathbf{F}=0$.

Vamos supor que a força $\mathbf{F}$ seja derivada de um potencial $V$, como no caso dos sistemas unidimensionais que estudamos anteriormente. Ou seja,

$$
\mathbf{F}=-\nabla V
$$

em que $\nabla$ é o operador gradiente:

$$
\nabla V \rightarrow\left(\frac{\partial V}{\partial x^{1}}, \frac{\partial V}{\partial x^{2}}, \frac{\partial V}{\partial x^{3}}\right)
$$

Então, (??) resulta em

$$
\frac{d}{d t}\left(\frac{1}{2} m v^{2}\right)=\mathbf{F} \cdot \dot{\mathbf{x}}=-\dot{\mathbf{x}} \cdot \nabla V=-\frac{d V}{d t}+\frac{\partial V}{\partial t}
$$

Neste caso, como $\mathbf{F} \cdot \mathbf{v}=d K / d t$,

$$
\frac{d}{d t}(K+V)=\frac{\partial V}{\partial t}
$$

Portanto, se a força é derivada de um potencial e independente do tempo, temos uma constante de movimento,

$$
E=K+V, \quad \frac{d E}{d t}=0,
$$

que é a energia mecânica da partícula. 


\section{Trabalho}

A energia de uma partícula em interação pode mudar com o tempo quando a interação é modelada com um potencial dependente do tempo, mas existem casos em que, sequer, uma energia potencial pode ser definida. É o caso, por exemplo, dos sistemas dissipativos, em que a energia cinética se perde através de outras formas de energia. O atrito, por exemplo, é um tipo de interação dissipativa, em que a energia do corpo é dissipada através do calor, de ondas sonoras ou, até, através de ondas eletromagnéticas. As interacões mecânicas no nosso ambiente são, com raríssimas exceções, dissipativas. Contudo, muitos sistemas desse tipo podem ser tratados com potenciais especiais, assumindo-se o conhecimento de um segundo sistema, de modo que o sistema físico de interesse e este sistema interagente formam um único sistema isolado.

Os sistemas verdadeiramente conservativos, surpreendentemente, tornam-se maioria quando tratamos de sistemas moleculares, atômicos e sub-atômicos, ou quando tratamos de interações em larga escala no Universo.

Ainda assim, há um conceito que descreve o quanto determinado sistema troca energia com o ambiente, quando sua energia cinética não é conservada. É o conceito de trabalho.

Vamos supor uma única partícula de massa $m$ que desenvolve uma curva $C_{1}$ em $\mathbb{R}^{3}$, como na primeira figura (1).
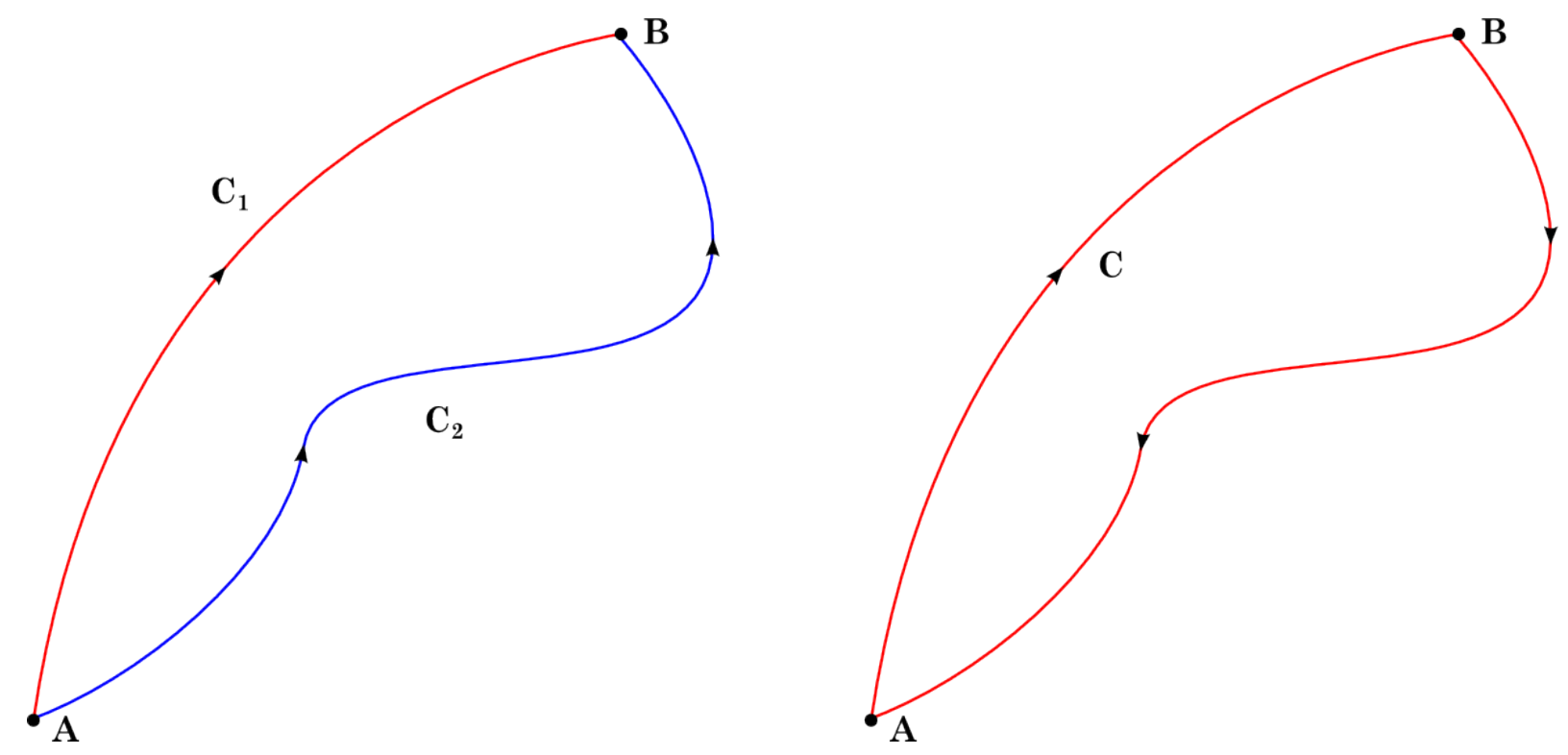

Figure 1: Na esquerda, uma partícula pode desenvolver uma curva $C_{1}$, ou uma curva $C_{2}$ entre os pontos $A$ e $B$. À direita, a partícula percorre a curva $C_{1}$ de $A$ a $B$, retornando ao onto $A$ através da trajetória $C_{2}$, formando um circuito fechado $C$.

Como vimos, no geral, a derivada temporal da energia cinética da partícula não deve ser zero. Temos que

$$
\frac{d K}{d t}=\mathbf{F} \cdot \dot{\mathbf{x}},
$$

o que vale em cada ponto da trajetória. Como a velocidade é tanjente a $C_{1}$ em cada ponto, o produto escalar à direita de (??) é o módulo da projeção ortogonal da força que age sobre a partícula, sobre sua velocidade. Este observável escalar é conhecido como Potência. 
A equação (??) pode ser escrita como

$$
d K=\mathbf{F} \cdot \frac{d \mathbf{x}}{d t} d t=\mathbf{F} \cdot d \mathbf{x}
$$

o que fornece a diferença infinitesimal da energia cinética relacionada à potência dissipada em uma uma porção infinitesimal de tempo (primeira igualdade), ou simplesmente à projeção da força sobre um elemento infinitesimal de distância sobre a curva (segunda igualdade). Esta equação nos fornece o quanto a partícula troca energia com o ambiente enquanto passa o tempo, sobre sua curva horária, fornecendo uma boa forma de definição de trabalho.

Agora, do ponto $A$ ao ponto $B$ sobre $C_{1}$, podemos integrar (??):

$$
W_{C_{1}} \equiv \int_{C_{1}} d K=\int_{\mathbf{x}_{A}}^{\mathbf{x}_{B}} \mathbf{F} \cdot d \mathbf{x}=\int_{t_{A}}^{t_{B}} \mathbf{F} \cdot \mathbf{v} d t
$$

Esta integral é definida como o trabalho da força $\mathbf{F}$ sobre a partícula entre os pontos $A$ e $B$ sobre a curva $C_{1}$. Note que a integral em $d \mathbf{x}$ é uma integral de linha.

Note que, em razão da expressão (??), o trabalho sobre a curva $C_{1}$ é dado por

$$
W_{C_{1}}=\int_{t_{A}}^{t_{B}} \mathbf{F} \cdot \mathbf{v} d t=\int_{t_{A}}^{t_{B}} d K=K\left(t_{B}\right)-K\left(t_{A}\right) .
$$

Portanto, o trabalho sobre uma curva é igual à variação total da energia cinética sobre a curva, $W=\Delta K$. Este resultado é conhecido como Teorema Trabalho - Energia Cinética.

Como toda integral de linha, o trabalho depende da curva seguida pela partícula. Isto deve ser evidente através da forma $\int_{C_{1}} \mathbf{F} \cdot d \mathbf{x}$, mas também pode ser visto na integral $\int_{t_{A}}^{t_{B}} \mathbf{F} \cdot \mathbf{v} d t$, na qual a curva deve estar codificada no campo vetorial $\mathbf{v}$. Assim, na primeira figura 1, o trabalho sobre a partícula para percorrer $C_{1}$ deve ser distinto do trabalho para percorrer $C_{2}$. Embora a partícula esteja submetida ao mesmo campo de forças $F(t, \mathbf{x}, \mathbf{v}, \cdots)$, a força em cada ponto da primeira curva será diferente da força em cada ponto da segunda curva, e o mesmo ocorrerá com o campo de velocidades. É natural não esperar que o resultado seja o mesmo.

\section{Forças conservativas}

Contudo, existem forças especiais, denominadas conservativas, cujo trabalho não depende da curva tomada pela partícula. A existência de forças conservativas é de fundamental importância para a validade da lei de conservação da energia, como veremos. A primeira consequência do fato de uma força ser conservativa é que, no exemplo da primeira figura 1 , o trabalho realizado sobre a partícula na curva $C_{1}$ é igual ao trabalho sobre a curva $C_{2}$, ou seja,

$$
\int_{C_{1}} \mathbf{F} \cdot d \mathbf{x}=\int_{C_{2}} \mathbf{F} \cdot d \mathbf{x}, \quad \text { ou } \quad \int_{C_{1}} \mathbf{F} \cdot d \mathbf{x}-\int_{C_{2}} \mathbf{F} \cdot d \mathbf{x}=0
$$

Em ambas as curvas, a partícula caminha do ponto $A$ ao ponto $B$. Porém, o que ocorre se, como na figura 1 à direita, a partícula volta pelo sentido oposto da curva $C_{2}$ após chegar ao ponto $B$ pela curva $C_{1}$ ? Neste caso, a partícula percorrerá um circuito fechado $C$ composto por $C_{1}$ e por $-C_{2}$, em que o sinal de menos significa a inversão da orientação da curva. Na integral de linha, a mudança de orientação de uma curva implica na mudança de sinal da integral, ou seja,

$$
\int_{-C_{2}} \mathbf{F} \cdot d \mathbf{x}=-\int_{C_{2}} \mathbf{F} \cdot d \mathbf{x}
$$


que resulta, caso a força seja conservativa, em

$$
\int_{C_{1}} \mathbf{F} \cdot d \mathbf{x}+\int_{-C_{2}} \mathbf{F} \cdot d \mathbf{x} \equiv \oint_{C} \mathbf{F} \cdot d \mathbf{x}=0,
$$

em que $\oint$ simboliza integração em uma curva fechada. O resultado vale para qualquer curva fechada, as curva $C_{1}, C_{2}$ e $C$ nada possuem de especial, portanto, o trabalho de uma força conservativa sobre uma curva fechada é sempre nulo.

Na figura 2, temos uma outra curva fechada $\gamma$ genérica. Toda curva fechada é uma fronteira para uma infinidade de superfícies abertas, como por exemplo, a superfície $\Sigma$.

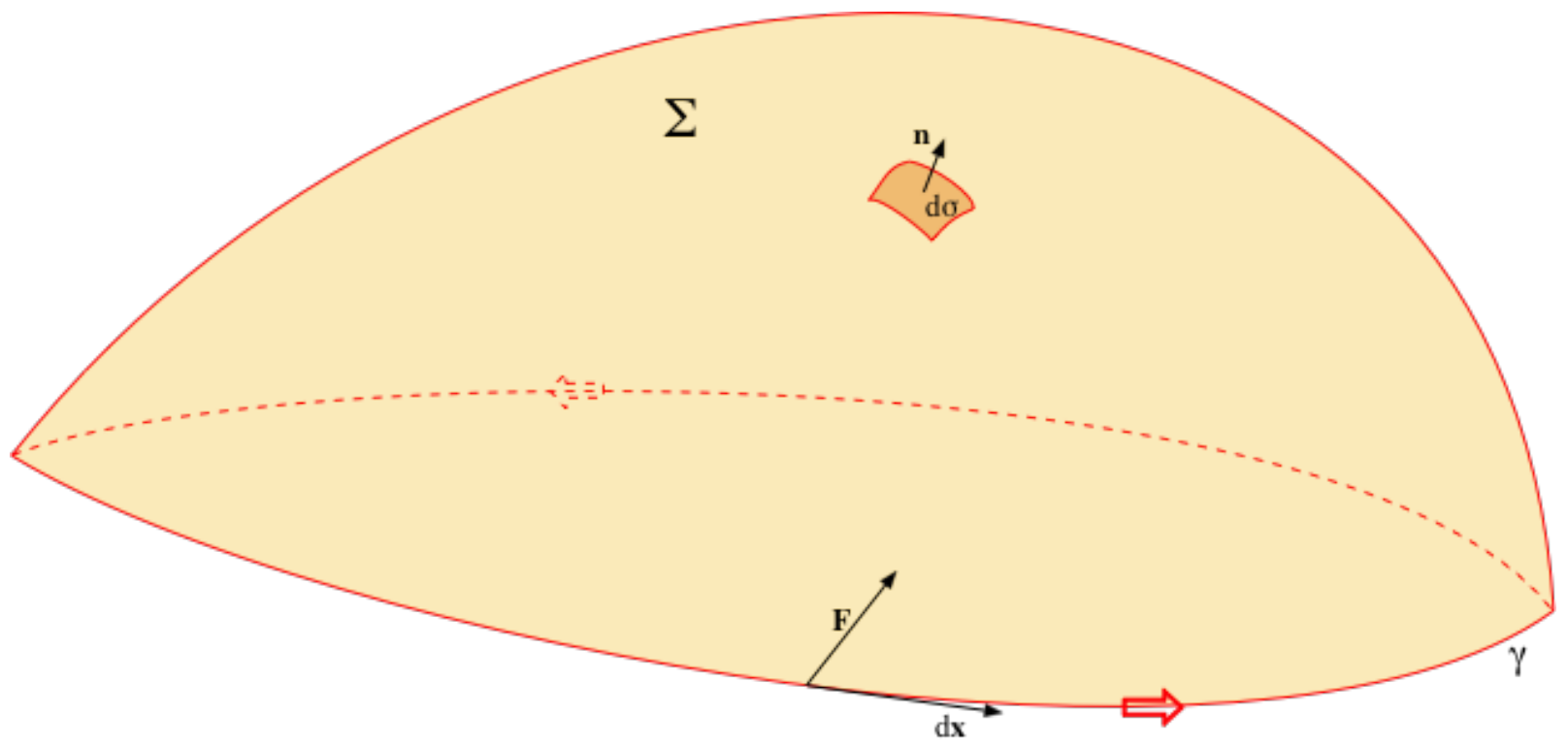

Figure 2: Palco geométrico para o teorema de Stokes. Uma curva $\gamma$ é fronteira para uma superfície $\Sigma$. Na curva, orientada no sentido anti-horário, $d \mathbf{x}$ é um elemento de linha diferencial, tangente a $\gamma$ em cada ponto, e um campo vetorial $\mathbf{F}$ é a força que age sobre uma partícula. Na superfície, cada ponto tem um vetor normal $\mathbf{n}$ ortogonal à um elemento de superfície $d \sigma$.

Para qualquer campo vetorial F, é válido o teorema de Stokes

$$
\oint_{\gamma} \mathbf{F} \cdot d \mathbf{x}=\int_{\Sigma} d \sigma \mathbf{n} \cdot \nabla \times \mathbf{F}
$$

em que a segunda integral é uma integral de superfície. O teorema de Stokes tem a interpretação de relacionar a circulação de um campo vetorial, $\oint_{\gamma} \mathbf{F} \cdot d \mathbf{x}$, ao fluxo de seu rotacional sobre uma superfície.

No nosso caso, a circulação de um campo de forças sobre uma curva fechada é exatamente o trabalho realizado sobre a partícula que percorre esta curva. Assim, se a força é conservativa, este trabalho é nulo, o que resulta em

$$
\int_{\Sigma} d \sigma \mathbf{n} \cdot \nabla \times \mathbf{F}=0
$$

Agora, é sempre possível que o rotacional de um campo vetorial seja ortogonal a determinada superfície $\Sigma$, mas o teorema de Stokes deve ser verdade para qualquer superfície encerrada pela curva. Assim, a igualdade acima resulta em

$$
\nabla \times \mathbf{F}=0
$$


ou seja, se $\mathbf{F}$ é conservativo, deve também ser um campo irrotacional.

Outro importante resultado, embora complicado de se demonstrar, é conhecido como teorema de Helmholtz. Este teorema nos diz que, sob certas condições de existência com as quais não nos preocuparemos, um campo vetorial $\mathbf{F}$ pode ser escrito por

$$
\mathbf{F}=-\nabla V+\nabla \times \mathbf{W}
$$

ou seja, pode ser decomposto em um gradiente e um rotacional. Note que as seguintes relações são, portanto, verdadeiras:

$$
\nabla \cdot \mathbf{F}=-\nabla^{2} V, \quad \nabla \times \mathbf{F}=\nabla \times \nabla \times \mathbf{W}
$$

já que o rotacional de um gradiente, assim como o divergente de um rotacional, são sempre nulos. Portanto, se a força é conservativa, temos a validade da equação (??), que resulta em

$$
\nabla \times \nabla \times \mathbf{W}=0 .
$$

A seguinte identidade é válida:

$$
\nabla \times \nabla \times \mathbf{W}=\nabla(\nabla \cdot \mathbf{W})-\nabla^{2} \mathbf{W}
$$

mas ela só será zero se $\nabla \times \mathbf{W}$ for igual ao vetor nulo. Neste caso,

$$
\nabla \times \mathbf{F}=0 \quad \Longrightarrow \quad \mathbf{F}=-\nabla V .
$$

No geral, $V$ é denomindado potencial, mas no nosso caso, veremos que esta função coincide com a energia potencial.

Assim, todas as afirmativas abaixo são equivalentes:

1. A força $\mathbf{F}$ é uma força conservativa.

2. O trabalho da força $\mathbf{F}$ não depende da curva.

3. O trabalho da força $\mathbf{F}$ depende apenas dos pontos inicial e final da curva.

4. O trabalho da força $\mathbf{F}$ sobre toda curva fechada é igual a zero.

5. O rotaçional da força $\mathbf{F}$ é igual a zero.

6. A força $\mathbf{F}$ é igual ao negativo do gradiente de um potencial.

\section{Novamente, conservação da energia}

Vamos supor uma força conservativa $\mathbf{F}$, que em razão dos desenvolvimentos anteriores, é igual ao negativo do gradiente de um potencial $V$. Vamos supor o caso em que o potencial depende apenas do tempo e da posição da partícula, por simplicidade. Veremos mais adiante que um potencial mais complicado, no geral, não será conservativo.

Neste caso, vimos que o trabalho desta força sobre uma curva qualquer é dada por

$$
W_{\gamma}=\int_{\gamma} \mathbf{F} \cdot d \mathbf{x}=-\int_{\gamma} d \mathbf{x} \cdot \nabla V,
$$

que vimos ser, também, igual à variação da energia cinética da partícula, ou seja,

$$
K\left(\mathbf{x}_{f}\right)-K\left(\mathbf{x}_{0}\right)=-\int_{\gamma} d \mathbf{x} \cdot \nabla V
$$

Note, contudo, que

$$
d V=\frac{\partial V}{\partial t} d t+\frac{\partial V}{\partial x} d x+\frac{\partial V}{\partial y} d y+\frac{\partial V}{\partial z} d z=\frac{\partial V}{\partial t} d t+d \mathbf{x} \cdot \nabla V
$$


em um sistema de coordenadas cartesiano. Neste caso,

$$
K\left(\mathbf{x}_{f}\right)-K\left(\mathbf{x}_{0}\right)=-\int_{\gamma} d V+\int_{\gamma} d t \frac{\partial V}{\partial t},
$$

que resulta em

$$
K\left(\mathbf{x}_{f}\right)-K\left(\mathbf{x}_{0}\right)=-V\left(\mathbf{x}_{f}\right)+V\left(\mathbf{x}_{0}\right)+\int_{\gamma} d t \frac{\partial V}{\partial t}
$$

ou,

$$
K\left(\mathbf{x}_{f}\right)+V\left(\mathbf{x}_{f}\right)=K\left(\mathbf{x}_{0}\right)+V\left(\mathbf{x}_{0}\right)+\int_{\gamma} d t \frac{\partial V}{\partial t}
$$

Aqui, temos a energia mecânica $E=K+V$, assim,

$$
E\left(\mathbf{x}_{f}\right)=E\left(\mathbf{x}_{0}\right)+\int_{\gamma} d t \frac{\partial V}{\partial t} .
$$

Se $\partial V / \partial t=0$, temos que $E\left(\mathbf{x}_{f}\right)=E\left(\mathbf{x}_{0}\right)$, o que é uma forma global de conservação da energia mecânica. Assim, forças conservativas conservam a energia mecânica em potenciais independentes do tempo. 